\title{
Missed opportunities for immunisation in health facilities in Cape Town, South Africa
}

\author{
N Jacob, MB ChB; D Coetzee, FCPHM \\ Western Cape Government: Health, and School of Public Health and Family Medicine, Faculty of Health Sciences, University of Cape Town, \\ South Africa
}

Corresponding author: N Jacob (nasj11@yahoo.com)

\begin{abstract}
Background. Childhood immunisations are a cost-effective public health intervention for prevention of infectious diseases. Immunisation coverage is still suboptimal, however, which may result in disease outbreaks. Immunisation at every contact with a health facility is a strategy developed by the World Health Organization in order to improve immunisation coverage.

Objectives. To estimate the prevalence of missed opportunities for immunisation at different levels of healthcare in the Western Cape Province, South Africa, and assess factors associated with missed opportunities.

Methods. The study included a health facility-based cross-sectional exit survey of caregivers with children up to 5 years of age, followed by a qualitative exploration of staff attitudes towards immunisation.

Results. The overall prevalence of missed opportunities for immunisation was $4.6 \%$; $81.3 \%$ of caregivers brought Road-toHealth booklets (RTHBs) to consultations; and $56.0 \%$ of health workers asked to see the RTHBs during consultations. Children attending primary level facilities were significantly more likely to have their RTHBs requested than children attending a tertiary level facility. Lack of training and resources and heavy workloads were the main challenges reported at secondary/tertiary level facilities.

Conclusion. Missed opportunities for immunisation at health facilities in Cape Town were low, probably reflecting good immunisation coverage among children accessing health facilities. Increased health worker support, particularly at secondary/tertiary levels of care, is needed to improve the use of RTHBs to provide immunisation.
\end{abstract}

S Afr Med J 2015;105(11):917-921. DOI:10.7196/SAMJ.2015.v105i11.10194

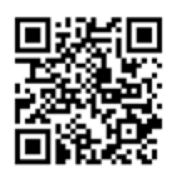

The Expanded Program on Immunization (EPI) initiated by the World Health Organization (WHO) in 1974 aimed to provide vaccines to children worldwide. $^{[1]}$ Despite advances in expanding immunisation services, coverage remains suboptimal in many areas. Where accessibility and utilisation of health services are low, every contact with a health facility provides an opportunity to immunise, particularly as these children are likely to be at an increased risk of vaccine-preventable diseases. ${ }^{[2,3]}$ The EPI Global Advisory Group ${ }^{[2]}$ defines a missed opportunity as 'any contact with a health service that did not result in an eligible child or woman receiving the needed vaccines. ${ }^{[1,2]}$ The elimination of missed opportunities can significantly improve immunisation coverage, thus reducing the risk of vaccine-preventable disease. ${ }^{[2]}$ The current immunisation schedule for the EPI in South Africa (EPI-SA) is in the Road-to-Health booklet (RTHB) issued to a child's mother at birth or to a subsequent caregiver.

Despite health facility immunisation coverage figures that exceed 95\%, the Western Cape Province (WC) of SA experienced a major measles outbreak in $2009 / 2010$. Herd immunity of $95 \%$ is required to prevent ongoing measles virus transmission. ${ }^{[4,5]}$ Low coverage, lower efficacy of some vaccines (e.g. measles vaccine at 9 months), incorrect vaccine administration and host response factors are the main causes of outbreaks of vaccine-preventable illness in areas with a functional immunisation programme. The 2009/2010 epidemic raised concerns regarding the validity of immunisation coverage data. Since coverage indicators are very sensitive to data inaccuracies such as incorrect population estimates, reported data may not be a true reflection of coverage in the population. In addition to improving the quality of coverage data, it is imperative that strategies to improve coverage are strengthened. The missed-opportunity survey was developed in 1984 to evaluate immunisation practices and improve immunisation coverage..$^{[1,3]}$

In 1991, the EPI reviewed all missed-opportunity studies published worldwide or reported to the WHO. ${ }^{[3]}$ Missed opportunities were found in all studies except one, with an overall median of $32 \%$ of children and women of childbearing age having had missed immunisation opportunities. ${ }^{[3]}$ Reasons for missed opportunities included false contraindications, health worker practices and vaccine shortages. A more recent systematic review by Rainey et al. ${ }^{[6]}$ evaluated reasons for under-vaccination of children in low- and middleincome countries. Immunisation system issues including missed opportunities, distance to services and low health worker knowledge were the most frequently observed reasons for under-vaccination. Missed-opportunity surveys conducted in the WC in the 1990s revealed a prevalence of $60-95 \%$. Category of consulting health worker, age of child and type of service (i.e. curative, preventive or integrated service) impacted on whether RTHBs were requested and immunisations given appropriately. . $^{[7-9]}$

A 2005 household survey among children aged 12 - 23 months in the WC revealed immunisation coverage rates of $76.8 \%$ for vaccines due by 9 months and $53.2 \%$ for vaccines due by 18 months. The main reasons for not being immunised were clinic-related factors, including missed opportunities (34\%). ${ }^{[10]}$ Studies conducted in developed countries have highlighted poor knowledge of EPI-SA, insufficient time, and staff not viewing immunisations as a priority or within their scope of practice. ${ }^{[11-13]}$ These factors have yet to be explored in developing countries. Few missed-opportunity studies have been conducted in the past decade, worldwide and in SA. 


\section{Methods}

A cross-sectional study design comprising two components was used:

1. A health facility-based cross-sectional survey to determine the prevalence of missed opportunities for immunisation and associated factors.

2. Qualitative exploration of staff attitudes towards immunisation using a semistructured questionnaire.

The study population was children $0-5$ years of age attending healthcare facilities with a caregiver from $08 \mathrm{~h} 00$ to $16 \mathrm{~h} 00$ on weekdays in the Cape Town metro.

Purposeful sampling was employed to select study sites. Five sites representative of primary, secondary and tertiary levels of care were selected, including a local clinic (clinic A - primary level), a community health centre (CHC B - primary level), one district hospital (hospital A -secondary level), one regional hospital (hospital B - secondary level) and one central hospital (hospital C tertiary level).

A sample size of 96 per facility was calculated, estimating that $50 \%$ of opportunities would be missed, with an alpha error of 0.05 and absolute precision of 0.1 . A recruiter identified caregiver/child pairs exiting the health facility, including both inpatients and outpatients. Only caregivers aged $>13$ years were included in the study. All caregivers were interviewed by a trained fieldworker. A request for an RTHB during the consultation was used as a proxy indicator that the immunisation status of a child was checked by the health worker.

Logistic regression was used to explore associations between outcomes (immunisation status, request for RTHB and presence of RTHB) and explanatory variables with adjustment for potential confounding variables. A forward selection procedure was applied for model building. The final model was selected by comparison of models using the likelihood ratio test and Akaike's information criterion.

In order to elicit themes regarding staff attitudes towards immunisation, a purposeful sample of two to three staff members at participating health facilities were interviewed by the primary researcher $(\mathrm{NJ})$ using a semistructured questionnaire. Data were analysed manually by NJ.

The research protocol was approved by the University of Cape Town Human Research Ethics Committee (HREC: 321/2014). The research followed the ethical standards outlined in the Helsinki Declaration $^{[14]}$ and the National Health Act. ${ }^{[15]}$ The risks to study participants were minimal.
Table 1. Descriptive characteristics of caregiver, child and visit to facility ${ }^{\star}$

\begin{tabular}{|c|c|}
\hline Variable & Overall \\
\hline Age of child (months), median (range) & $11(0-60)$ \\
\hline Age of caregiver (years) median (range) & $29(16-70)$ \\
\hline \multicolumn{2}{|l|}{ Day of week, \% (95\% CI) } \\
\hline Monday & $23.0(19.2-26.8)$ \\
\hline Tuesday & $19.1(15.6-22.6)$ \\
\hline Wednesday & $17.6(14.2-21.1)$ \\
\hline Thursday & $18.7(15.2-22.2)$ \\
\hline Friday & $21.6(17.9-25.3)$ \\
\hline \multicolumn{2}{|l|}{ Time of day, \% (95\% CI) } \\
\hline 09h00 - 11h59 & $41.8(37.4-46.3)$ \\
\hline $12 \mathrm{~h} 00-13 \mathrm{~h} 59$ & $32.3(28.0-36.5)$ \\
\hline $14 \mathrm{~h} 00-16 \mathrm{~h} 00$ & $25.9(21.9-29.9)$ \\
\hline Primary caregiver, $\%$ & 100.0 \\
\hline Specific illness reported by caregiver, \% ( $95 \% \mathrm{CI})$ & $21.6(17.9-25.3)$ \\
\hline HIV & $1.7(0.5-2.8)$ \\
\hline $\mathrm{TB}$ & $1.5(0.4-2.5)$ \\
\hline Malnutrition & $0.4(-0.2-1.0)^{\dagger}$ \\
\hline \multicolumn{2}{|l|}{ Health worker consulted, \% (95\% CI) } \\
\hline Doctor only & $39.2(34.8-43.7)$ \\
\hline Nurse only & $51.3(46.8-55.8)$ \\
\hline Doctor and nurse & $5.7(3.6-7.8)$ \\
\hline Allied health staff only & $3.8(2.1-5.5)$ \\
\hline RTHB asked for by health worker, \% (95\% CI) & $64.9(60.7-69.2)$ \\
\hline RTHB present, \% (95\% CI) & $81.3(77.8-84.8)$ \\
\hline \multicolumn{2}{|l|}{ Vaccines given today, $\%(95 \% \mathrm{CI})$} \\
\hline Yes - all pending vaccines given & $17.3(13.9-20.6)$ \\
\hline Yes - some pending vaccines given & $2.1(0.8-3.4)$ \\
\hline No & $80.7(77.1-84.2)$ \\
\hline \multicolumn{2}{|l|}{ Vaccine pending but contraindication to immunisation, $\%$ ( $95 \% \mathrm{CI})$} \\
\hline No & $4.6(2.7-6.4)$ \\
\hline Yes & $0.2(-0.2-0.6 \%)^{\dagger}$ \\
\hline Not applicable (complete immunisations) & $95.2(93.3-97.1)$ \\
\hline Immunisation status - complete by RTHB (N=392), \% (95\% CI) & $94.6(92.4-96.9)$ \\
\hline Immunisation status - complete by caregiver report $(N=90), \%(95 \% \mathrm{CI})$ & $86.7(79.5-93.8)$ \\
\hline \multicolumn{2}{|l|}{ Overall immunisation status, \% (95\% CI) } \\
\hline Complete by caregiver & $16.2(12.9-19.5)$ \\
\hline Uncertain by caregiver & $2.1(0.8-3.4)$ \\
\hline Complete by RTHB & $77.0(73.2-80.7)$ \\
\hline \multicolumn{2}{|l|}{ Missed opportunities by RTHB ( $N=21), \%(95 \% \mathrm{CI})$} \\
\hline Incomplete RTHB and checked by health worker & $61.9(39.3-84.6)$ \\
\hline \multicolumn{2}{|l|}{ Combined overall immunisation status, \% (95\% CI) } \\
\hline Complete (RTHB + caregiver) & $93.2(90.9-95.4)$ \\
\hline Uncertain (caregiver) & $2.1(0.8-3.4)$ \\
\hline Incomplete (RTHB + caregiver) & $4.6(2.6-6.4)$ \\
\hline Incomplete with contraindication & $0.2(-0.2-0.6)^{\dagger}$ \\
\hline $\begin{array}{l}\mathrm{CI}=\text { confidence interval. } \\
{ }^{*}=482 \text { unless specified. } \\
{ }^{N} \mathrm{Cls} \text { overlapping } 0 .\end{array}$ & \\
\hline
\end{tabular}


Participation in the study was voluntary, and all participants provided written informed consent. All children found to be eligible for immunisation were immunised on site in the designated clinical area. Verbal consent for immunisation was obtained from caregivers.

\section{Results}

Four hundred and eighty-two participants were recruited, with an overall respondent rate of $81.1 \%$. Respondent rates varied, ranging from $67.2 \%$ at hospital C to $86.4 \%$ at clinic A. Descriptive characteristics are summarised in Table 1.

The majority of children who participated in the study attended the facility for a consultation due to illness or for a follow-up consultation (Fig. 1). Discharged newborn infants exiting the facility were included, but no children discharged following inpatient admission participated in the study.

Of the caregivers, $81.3 \%$ had RTHBs present at the consultation. During children's consultations, $64.9 \%$ of health workers requested the RTHB. This decreased to $56.0 \%$ when excluding children who presented specifically for immunisation. There were notable differences between facilities. Only $11.6 \%$ of health workers requested to see RTHBs at hospital C, while $>70 \%$ of health workers at all other facilities requested the RTHB (Fig. 2). Of patients attending primary level facilities (clinic A and CHC B), $90.0 \%$ brought RTHBs to the facility. However, only $64.0 \%$ of patients attending hospital C did so.

Of the 392 children who had an RTHB present, $5.4 \%$ had incomplete immunisations. Of caregivers of the 90 children who did not have an RTHB present, $13.3 \%$ reported that immunisation status was incomplete or uncertain.

Overall, $77.0 \%$ of children had complete immunisations according to the RTHB, $16.2 \%$ had complete immunisations according to the caregiver's report, and the remaining $6.9 \%$ had incomplete immunisation status by RTHB or caregiver report or uncertain immunisation status by caregiver report. Of the 21 children with incomplete immunisations by RTHB, $61.9 \%$ had their RTHBs checked on the day, and $61.5 \%$ of these children received some, but not all, due immunisations on the same day. No facilities experienced vaccine stock-outs during the study period, and one child was erroneously identified by the health worker as too sick for immunisation. Only one child had a true contraindication to immunisation. The overall prevalence of missed opportunities for immunisation according to both RTHB and caregiver reports was $4.6 \%$. This figure increased to $6.6 \%$ when uncertain immunisation status was included. At all facilities, among children with RTHBs, $>90 \%$ of children exiting the facilities had complete immunisations required for age.

When excluding children presenting specifically for immunisation, $68.7 \%$ of children seen by nurses only had their RTHB requested, compared with $49.2 \%$ seen by doctors only.

The logistic regression revealed no statistically significant determinants of complete immunisation status. A number of factors associated with health worker requests for RTHB were identified (Table 2).

The model excluded those attending for immunisations and newborns who had been discharged. Similarly, none of the children accompanying a sick caregiver had RTHBs requested, and were also excluded from the model. Those with an RTHB present at consultation were 34.8 times more likely to have their RTHB requested by the health worker than those without RTHBs. A child presenting with an acute illness was 3.5 times more likely to have the RTHB requested compared with a child presenting for followup. Children presenting to health facilities from Monday to Thursday were more likely to have RTHBs requested than those presenting on Friday.

Children presenting to hospital C were least likely to have RTHBs requested. Those seen at clinic A were 17.2 times more likely to have their RTHBs requested than those seen at hospital C. Although exploratory

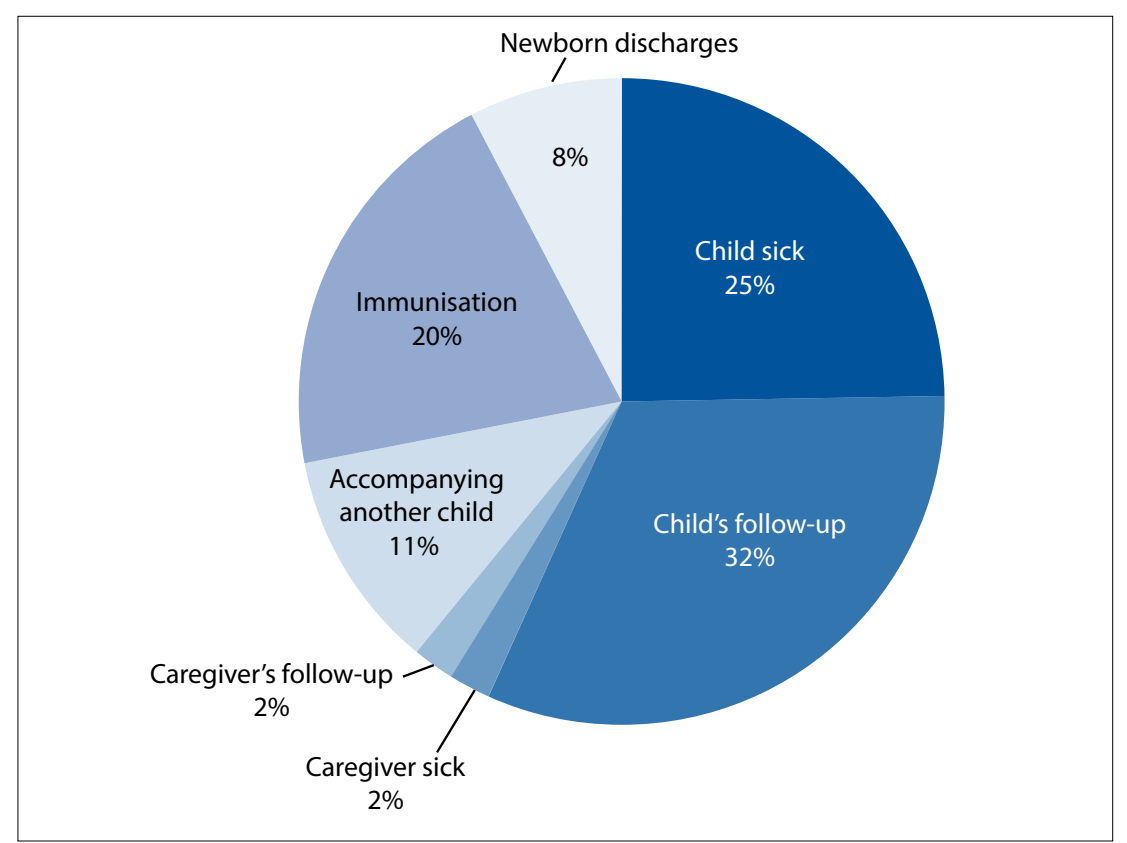

Fig. 1. Reason for attending health facility.

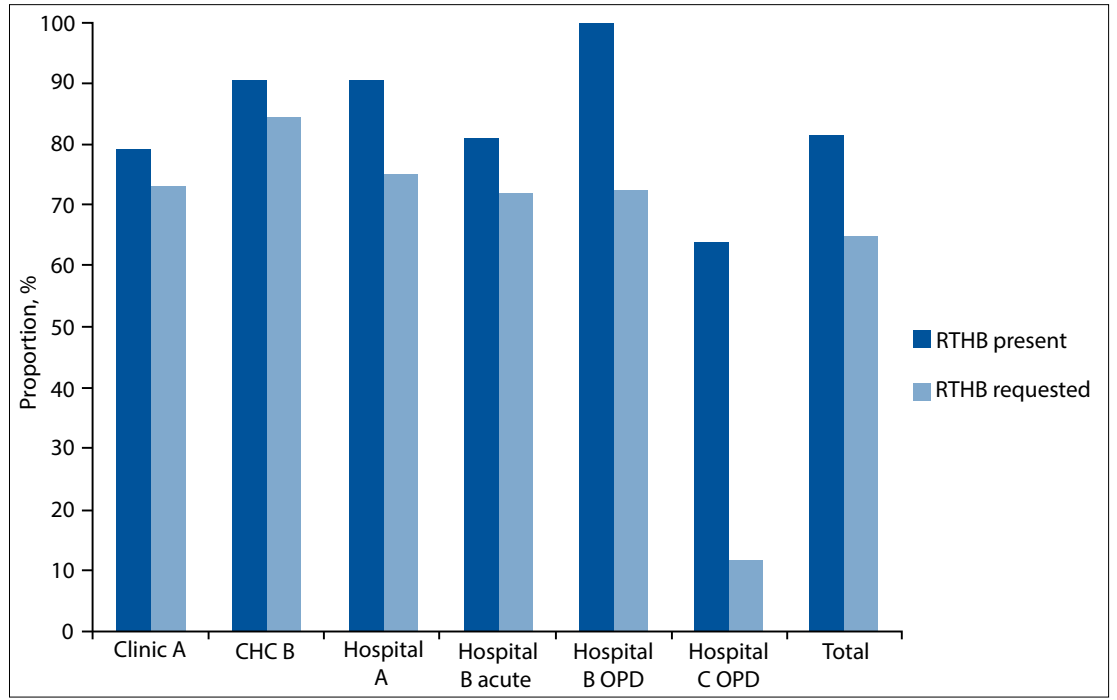

Fig. 2. RTHB requested by health worker, by facility. 
Table 2. Factors associated with health worker requests for RTHB

\begin{tabular}{|c|c|c|c|}
\hline Variable & OR & $p$-value ${ }^{\star}$ & 95\% CI \\
\hline RTHB present & 34.80 & 0.0000 & $7.32-165.43$ \\
\hline Age (months) & 0.97 & 0.0220 & $0.95-1.00$ \\
\hline \multicolumn{4}{|c|}{ Reason for attending (reference: child's follow-up) } \\
\hline Accompanying another child & 0.06 & 0.0000 & $0.01-0.26$ \\
\hline Caregiver follow-up & 0.09 & 0.1070 & $0.00-1.69$ \\
\hline Child sick & 3.49 & 0.0130 & $1.30-9.34$ \\
\hline \multicolumn{4}{|l|}{ Day (reference: Friday) } \\
\hline Monday & 1.66 & 0.4030 & $0.51-5.39$ \\
\hline Tuesday & 4.03 & 0.0790 & $0.85-19.05$ \\
\hline Wednesday & 15.44 & 0.0030 & $2.50-95.27$ \\
\hline Thursday & 11.10 & 0.0030 & $2.27-54.33$ \\
\hline \multicolumn{4}{|l|}{ Site (reference: hospital C) } \\
\hline Hospital A & 3.26 & 0.1950 & $0.54-19.55$ \\
\hline $\mathrm{CHC} \mathrm{B}$ & 7.31 & 0.0190 & $1.39-38.52$ \\
\hline Clinic A & 17.21 & 0.0000 & $4.52-65.42$ \\
\hline Hospital B acute & 1.46 & 0.7360 & $0.16-13.07$ \\
\hline Hospital B OPD & 5.34 & 0.1690 & $0.49-57.97$ \\
\hline
\end{tabular}

Table 3. Factors associated with RTHB present at health facility visit

\begin{tabular}{llll}
\hline Variable & OR & $p$-value & $\mathbf{9 5 \%}$ CI \\
\hline Reason for attending (reference: child's follow-up) & & & \\
$\quad$ Accompanying another child & 0.08 & $\mathbf{0 . 0 0 0 0}$ & $0.03-0.20$ \\
Caregiver follow-up & 0.04 & $\mathbf{0 . 0 0 1 0}$ & $0.00-0.26$ \\
Caregiver sick & 0.01 & $\mathbf{0 . 0 0 0 0}$ & $0.00-0.12$ \\
$\quad$ Child sick & 3.21 & $\mathbf{0 . 0 3 3 0}$ & $1.10-9.37$ \\
Age (months) & 0.96 & $\mathbf{0 . 0 0 0 0}$ & $0.95-0.98$ \\
Site (reference: hospital C) & & & \\
$\quad$ Hospital A & 5.52 & 0.0560 & $0.96-31.87$ \\
CHC B & 2.94 & 0.2140 & $0.54-16.14$ \\
$\quad$ Clinic A & 2.00 & 0.2120 & $0.67-5.95$ \\
$\quad$ Hospital B acute & 1.29 & 0.8090 & $0.16-10.43$ \\
Day (reference: Friday) & & & \\
$\quad$ Monday & 1.25 & 0.5890 & $0.56-2.78$ \\
$\quad$ Tuesday & 1.74 & 0.4310 & $0.44-6.92$ \\
$\quad$ Wednesday & 1.23 & 0.8250 & $0.20-7.54$ \\
$\quad$ Thursday & 1.21 & 0.8230 & $0.22-6.60$ \\
OR = odds ratio; CI = confidence interval. & & & \\
${ }^{*} p$-values $<0.05$ indicated in bold. & & &
\end{tabular}

analysis revealed that nurses were more likely than doctors to request RTHBs, this factor did not influence the model significantly, probably due to collinearity with site.

Younger children and those who were sick were significantly more likely to present with an RTHB, as shown in Table 3.

\section{Staff attitudes towards immunisation}

The majority of the 17 staff members interviewed 2 weeks after the quantitative component of the study said that they checked the RTHB and viewed it as an important and useful clinical tool. Doctors at tertiary level noted that RTHBs were less likely to be checked among follow-up patients, as they are well known to the hospital and assumed to be up to date with immunisations.

Many felt that a dedicated, well-trained immunisation nurse should be appointed at secondary/tertiary health facilities to prescribe and administer immunisations. The majority of those interviewed identified challenges that often led to missed immunisations at health facilities (Table 4)

\section{Discussion}

This study revealed that the prevalence of missed opportunities for immunisation at selected health facilities in Cape Town was low. The majority of children who presented specifically for immunisations received those immunisations on the day of the study, suggesting good local immunisation coverage among children accessing health facilities. However, children who do not access routine immunisation services are more likely to become ill, and to present particularly at secondary and tertiary services. The low percentage of health workers who requested RTHBs at these higher-level services indicates that vulnerable children could be missed. Furthermore, while a request for an RTHB was a proxy indicator for checking immunisation status, the health worker may have requested the RTHB to check other information.

A large proportion of children whose immunisations were incomplete had their RTHBs requested on the day and received some, but not all, of their immunisations. Although no vaccine stock-outs occurred during the study period and only one correct contraindication to immunisation was elicited, missed opportunities for immunisation may also be influenced by health worker knowledge regarding schedules and contraindications to immunisation. It appears that false contraindications to immunisation or concerns regarding simultaneous administration of immunisations contributed to the missed opportunities, as seen in similar studies. ${ }^{[3]}$

A number of factors were associated with requests for RTHBs by health workers during consultation. Having the RTHB present at the consultation had the largest effect. This may indicate that mothers were aware of the need to bring the RTHB at every visit, or that health workers were more likely to request the RTHB if it was visible to them at the consultation. The lower proportions of children with an RTHB present, as well as requests for an RTHB at hospital C, suggests that caregivers were aware that 
Table 4. Immunisation-related challenges

Vaccine stock-outs

Hospital pharmacies do not stock certain vaccines

Unavailability of vaccines after hours at all levels of care

Staff shortages and high workloads, particularly among nursing staff

Uncertainties among doctors regarding dosages and prescription format for immunisations

Pervasive nursing perspective that immunisations are only for primary level facilities

Poor staff training on immunisations, management of adverse events and cold-chain management

Staff conflict on appropriate hospital area where immunisations should be allocated

Lack of resources, e.g. EPI fridge

RTHBs were less likely to be utilised at facilities such as hospital C. Hospital C has more diverse patients attending follow-up services compared with the other facilities, and unmeasured contributory factors such as socioeconomic status and level of education may also have played a role. The main differences observed across health facilities were due to the nature of the visit. Secondary/tertiary level outpatient services typically see older children for follow-up purposes. At primary level facilities, younger children present for preventive care such as immunisation and management of acute illness. The markedly lower percentage of RTHBs requested in the hospital C outpatient department is probably due to the fact that these children are known to the doctors. Nevertheless, it reveals that routine documentation of health visits in the RTHB is practised infrequently. It also suggests that the RTHB is viewed as a tool for primary level only, with little relevance to tertiary facilities, and that it is not used to ensure good continuity of care across all levels.

Compared with other days, RTHBs were least likely to be requested on Mondays and Fridays, after adjustment for site, age of child and reason for attending the health facility. An increased patient load and health worker fatigue may have contributed to this finding.

Children accompanying other children for consultations and those accompanying caregivers for follow-up visits were markedly less likely than other children to have their RTHB requested. Nevertheless, some accompanying children had an RTHB with them. The presence of a child at any health facility is an opportunity for immunisation and health promotion, particularly where access to and utilisation of healthcare is poor.
A clear difference was seen in staff attitudes towards immunisation at secondary/ tertiary level facilities. Heavy workload, pharmacy stock practice, lack of training and uncertainty regarding immunisation guidelines and practices were cited as reasons for the avoidance of immunisation at hospitals. Clinicians preferred to refer to primary level facilities, creating a missed opportunity. Clinicians in secondary/tertiary facilities identified the need for a dedicated immunisation nurse who could administer immunisations appropriately and train other clinicians on guidelines and practices.

\section{Study limitations}

The poor response rate at hospital C may have introduced selection bias. Nonresponders were generally in a hurry and their children may have been less likely to be immunised. Only senior management at facilities was informed about the study, so that practices were not influenced by the study; however, awareness of the study over the study period may have influenced health worker practices. Furthermore, a number of questions in the questionnaire relied on caregiver recall. Social desirability bias may have influenced results, particularly when the RTHB was not presented. Extending the study after working hours would also have explored the prevalence of missed opportunities after hours, when resources, including time and staff, are often limited further. A household survey to identify missed opportunities would have been more representative, but also far more costly.

\section{Conclusion}

This study revealed a low prevalence of missed opportunities for immunisation at selected health facilities in Cape Town, reflecting good local immunisation coverage among children accessing the facilities. The lower proportion of health workers assessing RTHBs during consultations indicates that missed opportunities may occur if immunisation coverage is poor. Increased health worker support regarding immunisations is needed to ensure that opportunities for immunisation are not missed and immunisation coverage is improved still further.

Funding. We gratefully acknowledge the Western Cape Government: Health and the School of Public Health and Family Medicine, University of Cape Town, for their support in funding this study.

\section{References}

1. World Health Organization. Systematic review of missed opportunities for vaccination: Request for proposals. http://www.who.int/immunization/rfp_review_missed_ opportunities_vaccination/en/ (accessed 12 February 2014)

2. United Nations. Millennium Development Goals. http://www. un.org/millenniumgoals/ (accessed 12 February 2014).

3. Hutchins SS, Jansen H, Robertson SE, Evans P, Kim-Farley RJ. Studies of missed opportunities for immunization in developing and industrialized countries. Bull World Health Organ 1993;71(5):549-560

4. Nelson KE, Williams CM. Infectious Disease Epidemiology, Theory and Practice. Sudbury, MA: Jones \& Bartlett, 2006.

5. Bernhardt GL, Cameron NA, Willems B, Boulle A, Coetzee D.

Bernharde GL, Cameron NA, Willens B, Boulle A, Coetzee D. Evaluating measles vaccination coverage in high-incidence areas or the Western Cape Province, following the mass vaccination campaign. S Afr Med ) 2013;301(3):181-186. [http://dx.doi. org/10.7196/SAMJ.6196]

6. Rainey JJ, Watkins M, Ryman TK, Sandhu P, Bo A, Banerjee K. Reasons related to non-vaccination and under-vaccination of children in low and middle income countries: Findings from a systematic review of the published literature, 1999-2009. Vaccine 2011;29(46):8215-8221. [http://dx.doi.org/http://dx.doi. org/10.1016/j.vaccine.2011.08.096

7. Yach D, Metcalf C, Lachman P, et al. Missed opportunities for measles immunisation in selected Western Cape hospitals. S Afr Med J 1991;79(8):437-439.

8. Harrison D, Barron P, Glass B, Sonday S, van der Heyde Y. Far fewer missed opportunities for immunisation in an integrated child health service. S Afr Med J 1993;83(8):575-576.

9. Bachmann MO, Barron P. Missed opportunities for Bachmann MO, Barron P. Missed opportunities for
immunisation in curative and preventive services in a community health centre. S Afr Med J 1996;86(8):947-949.

10. Corrigall J, Coetzee D, Cameron N. Is the Western Cape at risk for an outbreak of preventable childhood diseases? Lessons from an evaluation of routine immunisation coverage. S Afr Med J 2008;98(1):41-45.

11. Prislin R, Sawyer MH, De Guire M, Brennan J, Holcomb K, Nader PR. Missed opportunities to immunize: Psychosocial and practice correlates. Am J Prev Med 2002;22(3):165-169. [http:// dx.doi.org/10.1016/s0749-3797(01)00429-9]

2. Prislin R, Sawyer MH, Nader PR, Goerlitz M, de Guire M, Ho S. Provider-staff discrepancies in reported immunization knowledge and practices. Prev Med 2002:34(5):554-561. [http $/ /$ dx.doi.org/10.1006/pmed.2002.1019]

13. Szilagyi PG, Rodewald LE, Humiston SG, et al. Immunization Szilagyi PG, Rodewald LE, Humiston SG, et al. Immunization
practices of pediatricians and family physicians in the United States. Pediatrics 1994;94(4):517-523.

14. World Health Organization. Declaration of Helsinki. http:// www.who.int/bulletin/archives/79(4)373.pdf (accessed 5 March 2014)

15. South African Government. National Health Act 61 of 2003. http://www.gov.za/documents/download.php?f=68039 (accessed 5 March 2014).

Accepted 10 October 2015. 